\title{
NEW MILLIMETRE MEASUREMENTS AND THE RADIO TO X-RAY CONTINUA OF ACTIVE GALACTIC NUCLEI
}

\author{
A.Lawrence \\ School of Mathematical Sciences, Queen Mary College, \\ Mile End Rd, London E14NS, UK.
}

I am one of a large team studying an X-ray flux limited sample of $35 \mathrm{AGN}$, at radio (Unger et al 1987 MNRAS 228 521), IR (Ward et al 1987 ApJ 31574 and Carleton et al 1987 ApJ 318 595), optical-UV (Boisson et al in preparation), and X-ray (Turner PhD thesis, Leicester) wavelengths. A gap in the data which we have just started to fill is the millimetre region. (Lawrence, Ward, Elvis, Robson, Smith, Duncan, and Rowan-Robinson). In Jan/Feb 1988 we made measurements of twelve objects at 800 and 1100 micron, using the ROE/QMC bolometer, UKT14, on the new UK/Dutch/Canadian facility on Mauna Kea, the James Clerk Maxwell

Telescope, reaching 1 sigma sensitivity of $\sim 15-20 \mathrm{mJy}$, an order of magnitude improvement over previous data. The four radio loud objects measured were easily detected, as expected. These all have a strong blazar component, showing smooth but curved spectra over many decades, possibly log-Gaussian in form (Landau et al 1986 ApJ 308 78), or alternatively explicable by a small number of power-law components (Robson et al 1988 MNRAS in press). In any case, other evidence points to non-thermal radiation by a relativistically moving feature (high polarization, strong variability, superluminal motion). Eight radio quiet objects were measured, and upper limits only found, except for a possible four sigma detection of N2992. In all cases, the mm limits are far below the 100 micron IRAS fluxes. In four of the nearest objects, this is not too surprising, as fluxes are rising steeply throughout 12 to 100 micron, a sign that the IRAS data is dominated by cool interstellar dust emission ("cirrus") from the discs of the parent galaxies. However we can also say that any postulated power law component of spectral index $\sim 1$ dominating the near-IR, must become self-absorbed around $\sim 200$ micron if the mm limits are not to be exceeded. Four rather more interesting objects are shown in Fig. 1. Again, any underlying power-law component must be self-absorbed by $\sim 100$ micron, but is not clear that such a powerlaw is needed. N5506 and IC4329A have falling optical energy distributions, and large $\mathrm{H} \alpha / \mathrm{H} \beta$ ratios; on the other hand, the IR continuum lies well above the X-ray level, so there is good argument for absorption and re-radiation by dust. N4151, while flat through the near-IR-optical, has a large hump centred at 25 micron. Particularly important here are further new measurements by Engargiola et al (1987, ApJ in press),and Edelson et al (1988, preprint) which show the energy distribution to be falling so steeply from 155 to 438 micron that selfabsorbed synchrotron is ruled out in this region. In fact, the whole energy distribution from $\mathrm{mm}$ to UV can be modelled without a power law at all, as shown in Fig 2. This uses a starburst component (from Rowan-Robinson and Crawford 1988, MNRAS in press), hot dust represented by three greybodies at $200 \mathrm{~K}, 500 \mathrm{~K}$, and $1000 \mathrm{~K}$, starlight from a nuclear cusp, and a blackbody at 30,000K. Even MKN590, which at first sight looks like a power-law, can be modelled by similar components (Fig. 3). 

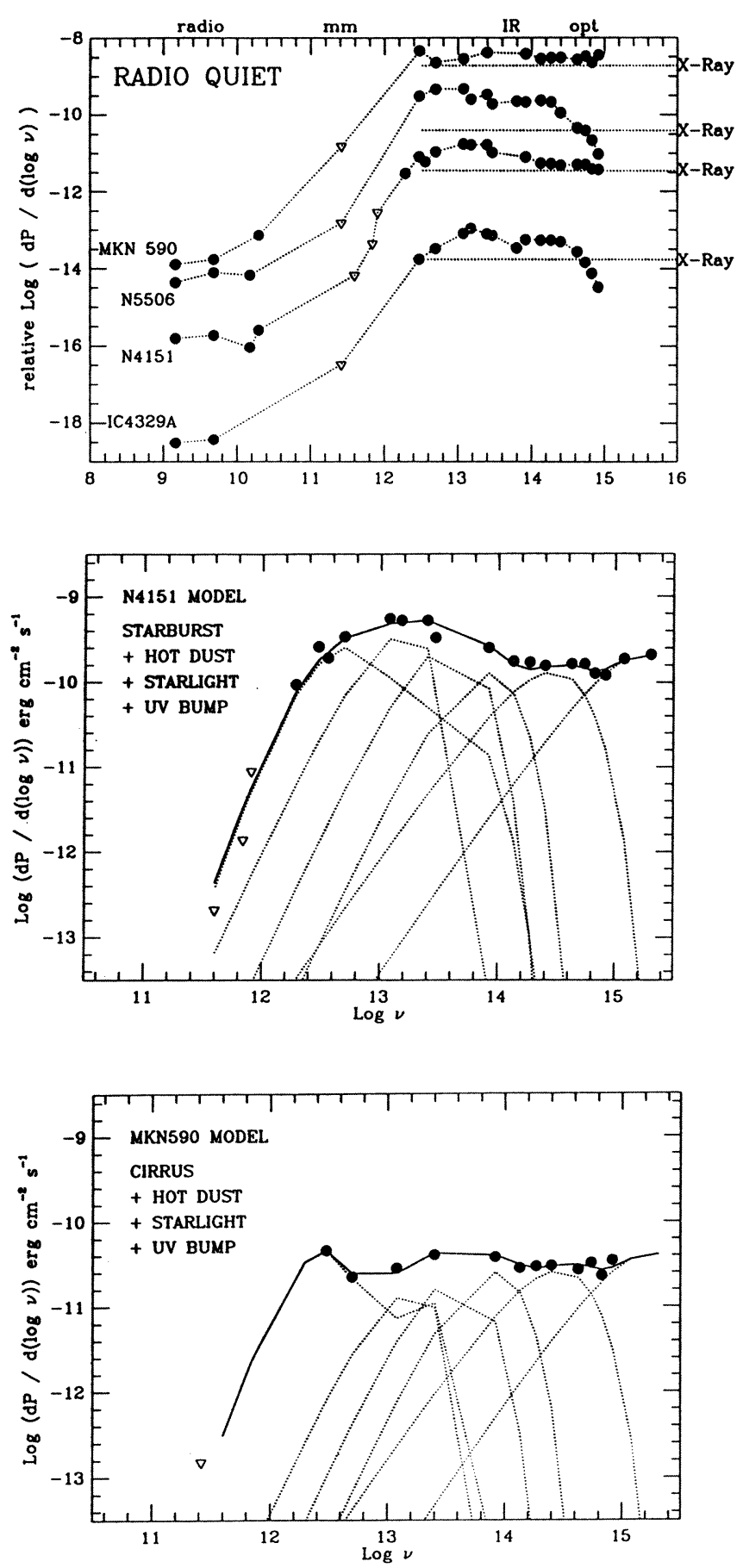


\section{DISCUSSION}

BIERMANN Our IRAM $1.3 \mathrm{~mm}$ orbservations have $1 \sigma \simeq 1 \mathrm{mJy}$.

LAWRENCE That's certainly very impressive.

MALKAN I'm still reluctant to jump on the hot dust bandwagon, at least as it applies to most luminous Seyfert 1 s and quasars. As B. Wills pointed out, they don't have much starlight, so that hot dust would produce a Wien cutoff by $1 \mu$, which has never been observed. Also, as you pointed out, the dust would need a covering fraction of at least $10-20 \%$ to produce the IR flux from absorption of the UV and X-rays. How could that be reconciled with the $X$-ray observations that virtually none of the high-luminosity objects show any absorption, even to extremely low levels of $N_{H}<$ a few $10^{20} \mathrm{~cm}^{20}$ ?

LAWRENCE I think it remains to be shown how much starlight there is in quasars near $1 \mu$. It was Soifer, not me, who claimed covering fractions of $10-20 \%$. I don't think we can answer this question in general as we don't know what happens to the spectrum in the EUV, and if the dust resides in gas of large optical depth, we have all the energy in the $X$-rays available as well. Anyway, whatever the covering fraction, the transition type $1 \mathrm{AGNs}$ are of course precisely those at which we are looking through a clear path.

B. WILLS The existence of power-law continua in lobe-dominant radio quasars and radio-quiet quasars ought to be seriously questioned as you have done. (There is a steep, $F_{\nu} \propto \nu^{-1.8}$, power law in most core-dominant radio quasars - see poster by D. Wills et al.). However, if you assume optically thick, geometrically thin accretion disk spectra to fit the blue-UV bump, you must have some explanation other than cool stars to contribute to the fit near $1 \mu$, because the cool stars will probably not contribute enough in the most luminous quasars.

LAWRENCE If a nuclear star cluster is intrinsic to the quasar phenomenon, there could be substantial starlight contribution even in high luminosity objects. In fact, however, it seemed to me looking at the quasars shown by Soifer earlier today, that these objects have a sharper inflection at $1 \mu$, and so presumably have a smaller starlight contribution. 